\title{
Turismo: Vieja y Nueva Gastronomía Iberoamericana y del Caribe
}

\begin{abstract}
Alfredo Ascanio ${ }^{1}$
RESUMO: La gastronomía local y vernácula es un elemento que forma parte del producto turístico orientado a nichos de mercados. Los recetarios de cocina, que esperan por ser investigados y rescatados, deben formar parte de una estrategia turística a ser institucionalizada como expresión cultural iberoamericana y del caribe. La investigación gastronómica y el desarrollo de una vieja y nueva cocina debe pertenecercomo proyecto a las Escuelas de Alta Cocina para formar los futuros chef de esta parte del continente americano.
\end{abstract}

PALABRAS CLA VES: gastronomía y turismo, recetarios de cocina, cocina vernácula, investigación gastronómica, Escuelas de Alta Cocina.

ABSTRACT: The local and typical gastronomy are components of the tourist product to special touristic places. The recipe books which are to be investigated and to be recovered must be part of a touristic strategy to be institutionalized as an Iberoamerican and Caribean cultural expressions. In this part of the American continent theold and new gastronomy investigation and development should be considered as a project in the "High Cooking Schools", where the future chefs will be prepared.

KEYWORDS: Gastronomy and tourism; recipe books; typical cooking; gastronomy investigation; High Cooking Schools.

1. Economista y profesor titular de la Universidad Simón Bolívar en Caracas - Venezuela. Postgrado en la Escuela de Negocios de Harvard y Maestría en Ciencias Políticas de la USB. Vice-Ministrode Turismo de Venezuela. Consejero al Secretario General de la OMT. E-mail: aascanio@usb.ve 


\section{Introducción}

La fabricación de productos turísticos novedosos mediante la incorporación de los actores de la propia comunidad es una necesidad urgente. Este proceso de la elaboración de un producto nuevo, se puede lograr incorporando al recurso local un valor agregado que no es infraestructural sino organizacional, pues de lo que se trata es poner en valor un atractivo que debe ser: accesible, aportado por una nueva cultura en instalaciones adecuadas; y mediante el servicio de un personal de contacto o anfitrión, que no sólo conoce lo que puedeaportaral turismo, sino que puede recibir también un adiestramiento nuevo para que complemente su antigua o moderna experticia (Monreal, 1998: 72-74).

Un elemento turístico local importante es la gastronomía orientada a segmentos del mercado turístico. El historiador chileno Roberto Paez nos recuerda que en el campo gastronómico muchas recetas son secretos celosamente guardados y difíciles de otorgarles una autoría; pero cuando nos relata el muestrario de la comida que se sirve a los turistas en el puerto de Coquimbo y en los balnearios de Guanaqueros y Tongoy, nombra de inmediato la albacora con salsa de mantequilla, la palometa con salsa lupa, el chupe de jaiba y los mariscales parmesanos.

Durante el siglo XIX en Chile existió la "belle epoque" gastronómica, debido a la influencia de los platos franceses e ingleses que se arraigaron a la par de la explotación minera, como por ejemplo: cl guiso de ostras a la John, que fue una recetaperdida en el tiempo (Paez, 1998: 105-110) y que si hoy fuese rescatadapodría sorprender a los turistas europeos.

En todos los países de Iberoamérica y del Caribe se ha trabajado el área de los recetarios de cocina con mucho interés y por mucho tiempo. Existen países como México, por ejemplo, que ha podido internacionalizar su platillos vernáculos. En otros países aún no se ha podido capitalizar un patrimonio tan importante como lo es el culinario.

\section{Recetarios Antiguos, Concursos Modernos y Recetarios Actualizados}

Los antiguos recetarios de la gastronomía latinoamericana hoy debieran ser un tesoro para las nuevas escuelas de alta cocina que se incorporan al turismo. Pocos recetarios se han podido conservar y muchos permanecen en archivos familiares. No obstante, es necesario rescatar, de los periódicos de la época, aquellas recetas que forman el verdadero patrimonio turístico gastronómico. Por ejemplo, en un diario provincial chileno de 1900, se difundía la receta del llamado filete a la coquimbana, un plato para lucirse ante invitados nacionales e internacionales (Paez, 1998:113).

Incluso los poetas chilenos que vivieron desde 1900 hasta 1960, como Pablo de Rokha,por ejemplo, seudónimo de Carlos Díaz Loyola, tuvieron la osadía de cantar la vida chilena sencilla en sus comidas y bebidas En el poema titulado Epopeya de las comidas y las bebidas de Chile, el poeta Rokha nos habla de camarón del Huasco y de la olorosa empanada colchagüina, así como del costillar de chancho con ajo, picantísimo, y la guañaca en caldo de ganso, acompañado con el vino de Pocoa (Rokla, 1992:191-211).

En una muy antigua guía turística denominada Venezuela: a land where it's always summer, su autor, el norteamericano WilliamEleroy Curtis (1896), se refería con entusiasmo acerca de la calidad del café y el cacao venezolano y en especial las variedad de café denominada "Caracas Washed" y las dos variedades de cacao llamadas "El Criollo" y "El Trinitario"; pero también, recientemente, la revista Pandora (Diario El Nacional), realizó un concurso de la nueva cocina venezolana y el 27 de enero de 1996 publicó las recetas ganadoras como la salchicha de hongos en salsa de perejil, un plato salado inventado por Carlos Becerra; y el paté Barlovento en crema de guayaba de Brizeida Marrón, así como doce platos más entre salados y dulces.

En Venezuela la cocina tradicional ha tomado prestigio por iniciativa de Armando Scannone, quien se ha dedicado a recopilar recetas con énfasis en las que provienen del entorno de Caracas y su sazón. No obstante, la cocina del país como la pizca andina, los platos zulianos a base de coco, la cocina llanera de cacería, la que proviene de la Guayana Venezolana a base del merey, y el llamado talkarí oriental, se pueden encontrar en el libro Geografía Gastronómica Venezolana de Ramón David León , pues allí aparece la verdadera cocina de origen doméstica desarrollada en los hogares.

En el caso uruguayo, el Manual de Cocina (1965) del Instituto Crandon tuvo su origen en las pequeñas escuelas de economía doméstica fundadas por Cecilia Güelfi en el año de 1879, de allí que su énfasis fue siempre el valor nutritivo de los alimentos y la dieta semanal de por lo menos 1.100 calorías al día, sin olvidar el aspecto atractivo del arreglo de la mesa, el planeamiento de menúes, el equipo para la cocina, los envases para las medidas de ingredientes, la definición de los términos usados y la conservación de los alimentos.

\section{Una Labor de Años para ser Instifucionalizada}

Los ejemplos anteriores no es más que una muy pequeña muestra de la labor tesonera que por años muchas personas, unas sin fines de lucro y otras con un marcado interés comercial, han venido trabajando el área de los recetarios de la cocina latinoamericana. Sinembargo todavía las grandes cadenas hoteleras no aprecian en todo su valor la cocina vernácula, sino la cocina internacional promovida por la mayoría de los Chef europeos que contratan.

El turismo gastronómico se fundamenta más bien en la cocina tradicional de cada país; una cocina que nació en los hogares y que es también muchas veces una cocina mestiza donde lo ibérico, lo indígena y lo africano producen una fórmula 
mágica que logra una sazón diferente en cada espacio geográfico. Incluso, en algunos de nuestros países como México, Perú y Bolivia la cocina autóctona de origen asteca maya e incaica, sí ha logrado un posicionamiento incluso a nivel internacional también la cocina típica de Bahía (Brasil), la cual cuenta con un mercado cautivo local que gasta más del $20 \%$ del presupuesto familiar dedicado a la demanda de bienes culturales (Burman, 1998:76); pero no es el caso de la gastronomía caribeña, por ejemplo, la cual no ha recibido el respaldo adecuado de las oficinas de turismo de la región.

Lo que ha faltado para que esa cocina se institucionalice, y pueda ser un recurso turístico de primer orden, es la búsqueda y rescate de recetas antiguas para poder utilizarlas tal como eran o modernizarlas; la creación de Escuelas de Alta Cocina que puedan realizar una labor de investigación gastronómica y formación profesional ; y finalmente el reconocimiento de los entes nacionales de turismo de la importancia de la gastronomía tradicional y de la nueva gastronomía inventada localmente, como un patrimonio turístico.

\section{Una propuesta para Puebla}

Es conveniente que desde Puebla, México, un Estado y un País con una verdadera tradición culinaria, surja una iniciativa para que con el apoyo de la Secretaría de Turismo de Puebla y de la Unesco se pueda desarrollar una Escuela Iberoamericana y del Caribe de Alta Cocina, dedicada a la investigación gastronómica y a la innovación de nuevos platos, como expresión cultural de esta parte del continente americano.

En el Congreso de Puebla tienen que surgir recomendaciones vigorosas para que los organismos de turismo de los países iberoamericanos y del Caribe incorporen en su sistema turístico nacional no sólo los servicios de la restauración, que ya forman parte del sistema como empresas turísticas, sino las organizaciones privadas o bien las ONG dedicadas a la tarea integral de rescatar las antiguas recetas de la cocina latinoamericana y caribeña, de inventar nuevos platos, nuevos dulces y nuevas bebidas, con la idea de potenciar la oferta en los restaurantes de la región y otorgarles así una novedosa parcela de competitividad frente a la avasallante comercialización de la cocina internacional de origen europeo, norteamericana y asiática.

Una Escuela de Alta Cocina Iberoamericana y del Caribe dedicada a la formación de los futuros Chef de esta parte de continente, puede suscribir convenios de "joint venture" o de alianzas estratégicas con escuelas e institutos ya existentes que han explorado menos la cocina tradicional - con la excepción de algunos paísesy han abandonado la investigación culinaria; lo cual es un error, pues esta tarea es una manera estratégica de inventar nuestras rutas gastronómicas, que debería formar parte de los "paquetes turísticos" que elaboran los tour-operadores, ya que sin una adecuada comercialización de esa oferta, sería difícil que los restaurantes se inclinaran por añadir nuevos platilloscuando planifican sus menúes. Tiene que haber un segmento del mercado con poder adquisitivo discrecional, para que pueda ser rentable la incorporación de platos que han necesitado de la investigación y de un desarrollo previo.

Además, en una empresa de servicios alimenticios no se trata de vender sólo la comida que surgen de recetasexóticas, sino es necesario vender experiencia locual involucra atmósfera, decoración interior, mobiliario, uniformes del personal, atención cálida al cliente, todo ello como elementos sistémicos que, como objetivo, deben lograr la satisfacción del consumidor más exigente (Zancudo y Alcántara, 1999: 137-138).

\section{Referencias Bibliográficas}

BURMAN, Grazia. 1998. Impacto da cultura na economía da Bahía. Participação no gasto turístico e consumo de residentes e governo Turismo em Análise, ECA/USP, Brasil, v. 9, n. 2, p. 70-87.

ROKHA, Pablo de. 1991. Antologia. Madrid: Visor Libros.

EL NACIONAL, Revista Pandora. 1996. Recetas de concurso, Caracas: Venezuela, n. 670, enero.

INSTITUTO CRANDON. 1965. Manual de cocina del Instituto Crandon. Montevideo: Colombino Hermanos.

MONREAL, Eduardo. 1998. Desarrolloeconómico local desde una perspectiva turística. RUTA, Revista Universituria de Turismo y Administración, Chile Universidad de La Serena, n. 3. p. 69-81, $2^{\circ}$ Semestre

PAEZ, Roberto. 1998. De las ostras a lo John a la gastronomía coquimbana. RUTA, Revista Universituria de Turismo y Administración, Chile: Universidad de La Serena, n. 3. pp. 105-113, $2^{\circ}$ sem.

SCANNONE, Armando. 1986. Mi Cocina. A la munera de Caracas. 4 ed. Caracas: Jaimes Libros.

ZANCUDO, María Teresa; ALCANTARA, Maria Cris ${ }^{\top}$ na. 1999. Diseño estratégico de empresas de servicios alimenticios. Estudios y Perspectivas en Turismo, Bue. Ios Aires, CIET, v. 8, n. 1 y 2, p. $135-147$

\section{Recebido em 20/7/99}

Aprovado em 12/8/99 